\title{
TORSION-FREE GROUPS AND AMALGAMATED PRODUCTS ${ }^{1}$
}

\author{
G. BAUMSLAG, A. KARRASS AND D. SOLITAR
}

\begin{abstract}
There exist continuously many finitely generated torsion-free groups which cannot be obtained from the infinite cyclic group by repeatedly forming the free product with an amalgamated subgroup of any two groups already obtained, taking subgroups, and forming ascending unions.
\end{abstract}

1. Let $\mathcal{C}$ be the class of those groups which can be obtained from the infinite cyclic group by repeatedly forming the generalized free product with an amalgamated subgroup of any two groups already obtained, taking subgroups and forming ascending unions. Then every group in $\mathcal{C}$ is torsion-free (see e.g. B. H. Neumann [7]). Recently Graham Higman has again raised the possibility that the converse is also true. However this is by no means the case for we shall prove the following

THEOREM. There exist continuously many finitely generated torsionfree groups which lie outside $\mathfrak{C}$.

Now it is easy to prove (e.g. by using the technique developed in G. Higman, B. H. Neumann and Hanna Neumann [3]) that if $A$ and $B$ lie in $\mathcal{C}$ so does their direct product $A \times B$ and also if $A$ is a normal subgroup of a group $G$, and if $G / A$ is infinite cyclic then $G \in \mathrm{e}$ whenever $A \in \mathcal{C}$. This suggests that a torsion-free finitely generated group $G$, whose factor derived group $G / G^{\prime}$ is finite, might well lie outside $\mathfrak{C}$, and indeed this is the motivating fact in a search for groups outside $\mathcal{C}$.

2. Let us put $\mathcal{C}_{0}=\{Z\}$ and define for each ordinal $\alpha$ a class of groups $\mathfrak{C}_{\alpha}$ as follows: If $\alpha=\beta+1$ then $\mathfrak{C}_{\alpha}$ consists of the subgroups of any free product with an amalgamated subgroup of two groups in $\mathbb{C}_{\beta}$; if $\alpha$ is a limit ordinal then $\mathfrak{C}_{\alpha}$ is the class of groups which are the union of an ascending chain of groups, one from each $\mathfrak{C}_{\beta}$ with $\beta<\alpha$. Thus $\mathfrak{C}$ is the union of the $\mathcal{C}_{\alpha}$. We observe that each $\mathcal{C}_{\alpha}$ is closed under the formation of subgroups.

Received by the editors September 22, 1969.

AMS Subject Classifications. Primary 2052, 2010; Secondary 2018, 2027.

Key Words and Phrases. Torsion-free groups, amalgamated products, generalized free products, presentations, Reidemeister-Schreier theory, indecomposable subgroups, subgroup structure.

1 This research was supported in part by a grant from the National Research Council of Canada. 
The following lemma is crucial in our proof that $\mathfrak{e}$ does not contain all torsion-free groups.

LEMMA 1. Let $G$ be a finitely generated group which is indecomposable with respect to the free product with an amalgamated subgroup, i.e., if

$$
G=\left(A^{*} B ; U\right)
$$

then either $A=U$ or $B=U$. If $G / G^{\prime}$ is finite and $G \in \mathbb{e}$ then $G$ is the identity group.

Proof. Suppose $G \in \mathfrak{e}, G \neq 1$. Let $\alpha$ be the least ordinal for which $G \in \mathfrak{C}_{\alpha}$. Since $G$ is finitely generated (and hence not the union of a properly ascending infinite chain of subgroups) $\alpha$ is not a limit ordinal. Therefore $\alpha=\beta+1$ for some ordinal $\beta$. This means that $G$ is a subgroup of $P=(A * B ; U)$ for a suitable choice of $A, B \in \mathfrak{C}_{\beta}$. But, by Theorem 6 of [4], every finitely generated indecomposable subgroup of $P$ either has an infinite cyclic factor group or else is contained in a conjugate of $A$ or $B$. So $G$ is isomorphic to a subgroup of $A$ or $B$ and therefore $G \in \mathfrak{C}_{\beta}$. This contradicts the minimality of $\alpha$. Hence $G=1$ as claimed.

3. We are now in a position to construct our torsion-free groups which lie outside $\mathcal{C}$. To this end let $F$ be the free nilpotent group of class two on $a$ and $b$. Thus

$$
F=(a, b ;[a, b] a=a[a, b], b[a, b]=[a, b] b)
$$

where $[a, b]=a^{-1} b^{-1} a b$. Put $c=[a, b]$. The mapping

$$
a \rightarrow b^{-1} a^{-1}, \quad b \rightarrow a
$$

defines an automorphism $\phi$ of $F$ of order three which leaves $c$ fixed. We define (cf. J. F. Bowers [1])

$$
\begin{aligned}
G=(a, b, t ;[a, b] a=a[a, b], b[a, b] & =[a, b] b, t^{-1} a t \\
& \left.=b^{-1} a^{-1}, t^{-1} b t=a, t^{9}=[a, b]\right) .
\end{aligned}
$$

Then it is easy to see, either by using the classical extension theory of O. Schreier (see e.g. A. G. Kurošh [5]) or by making use of the presentation theory of Reidemeister and Schreier as described in [6], that $F$ is naturally embedded in $G$; and moreover, since $c$ has no cube root in $F$, that $G$ is torsion-free. But $G / G^{\prime}$ is of order 27. So if $G$ is indecomposable, then, by Lemma $1, G \notin \mathcal{C}$. But $G$ satisfies the identity

$$
\left[\left[x^{3}, y^{3}\right], x^{3}\right]=1 \text {. }
$$

On the other hand if $P=(A * B ; U)$ and $A \neq U \neq B$, then either $P$ 
contains a free subgroup of rank two, or $U$ is of index two in both $A$ and $B$. This means that a generalized free product $P$ never satisfies the identity (1).

Now there exist continuously many finitely generated torsionfree groups (P. Hall [2]). If $H$ is any one of these groups then of course $G \times H \notin \mathcal{C}$. Moreover it is not difficult to choose the groups $H$ so that the number of nonisomorphic groups $G \times H$ that arise is the power of the continum. This completes the proof of the theorem.

\section{REFERENCES}

1. J. F. Bowers, On composition series of polycyclic groups, J. London Math. Soc. 35 (1960), 433-444 MR 23 \#A1710.

2. P. Hall, Finiteness conditions for soluble groups, Proc. London Math. Soc. (3) 4 (1954), 419-436. MR 17, 344.

3. G. Higman, B. H. Neumann and Hanna Neumann, Embedding theorems for groups, J. London Math. Soc. 24 (1949), 247-254. MR 11, 322.

4. A. Karrass and D. Solitar, The subgroups of a free product of two groups with an amalgamated subgroup, Trans. Amer. Math. Soc. (to appear).

5. A. G. Kuroš, The theory of groups, 2nd ed., GITTL, Moscow, 1953; English transl., Chelsea, New York, 1955. MR 15, 501. MR 17, 124.

6. W. Magnus, A. Karrass and D. Solitar, Combinatorial group theory: Presentations of groups in terms of generators and relations, Pure and Appl. Math., vol. 13, Interscience, New York, 1966. MR 34 \#7617.

7. B. H. Neumann, An essay on free products of groups with amalgamations, Philos. Trans. Roy. Soc. London Ser. A. 246 (1954), 503-554. MR 16, 10.

RICE UNIVERSITY AND YORK UNIVERSITY 